\title{
Description of nighttime cough epochs in patients with stable COPD GOLD II-IV
}

This article was published in the following Dove Press journal: International Journal of COPD

\author{
Patrick Fischer' \\ Volker Gross' \\ Johannes Kroenig² \\ Andreas Weissflog ${ }^{3}$ \\ Olaf Hildebrandt ${ }^{2}$ \\ Keywan Sohrabi ${ }^{1,4}$ \\ Ulrich Koehler ${ }^{2}$ \\ 'Faculty of Health Sciences, \\ University of Applied Sciences, \\ Giessen, Germany; ${ }^{2}$ Department \\ of Pneumology, Intensive Care and \\ Sleep Medicine, University Hospital \\ of Marburg and Giessen, Marburg, \\ Germany; ${ }^{3}$ Clinical Research \\ Department, Thora Tech GmbH, \\ Giessen, Germany; ${ }^{4}$ Institute for \\ Medical Informatics, Justus-Liebig- \\ University Giessen, Giessen, Germany
}

Correspondence: Volker Gross Faculty of Health Sciences, University of Applied Sciences, Wiesenstrasse 14, D-35390 Giessen, Germany

Tel $+4964|309667|$

Fax +49 64I 3092920

Email volker.gross@ges.thm.de
Purpose: Chronic cough is one of the main symptoms of COPD. Ambulatory objective monitoring provides novel insights into the determinants and characteristics of nighttime cough in COPD.

Materials and methods: Nighttime cough was monitored objectively by LEOSound lung sound monitor in patients with stable COPD II-IV. In 30 patients, with 10 patients in each stage group, nighttime cough was analyzed for epoch frequency, epoch severity (epoch length and coughs per epoch), and pattern (productive or nonproductive).

Results: Cough was found in all patients ranging from 1 to 294 events over the recording period. In 29 patients, cough epochs were monitored, ranging from 1 to 75 epochs. The highest amount of cough epochs was found in patients with COPD stage III. Active smokers had significantly more productive cough epochs (61\%) than nonsmokers (24\%).

Conclusion: We found a high rate of nighttime cough epochs in patients with COPD, especially in those in stage III. Productive cough was predominantly found in patients with persistent smoking. LEOSound lung sound monitor offers a practical and valuable opportunity to evaluate cough objectively.

Keywords: cough, COPD, nighttime, cough-monitor, lung sound, cough epochs

\section{Introduction}

COPD represents a significant public health problem worldwide. COPD is characterized by chronic inflammation and irreversible airway obstruction. Common symptoms are breathlessness, chronic cough, wheezing, and chest tightness. Patients with chronic bronchitis and COPD suffer from chronic cough during daytime and nighttime. ${ }^{1}$ Cough and other symptoms of COPD differ in the morning and at night. ${ }^{2,3}$ Respiratory symptoms are particularly disruptive during night and early morning hours, contributing to sleep disturbance, limited morning activities, and poor health status. ${ }^{3,4}$ Productive cough is the strongest in the morning hours and is highly suggestive of COPD and reflects clearing of retained secretions. Epidemiological studies show a correlation between the progression of COPD and chronic cough with sputum production. In The Copenhagen City Heart Study, chronic mucus hypersecretion was significantly associated with greater $\mathrm{FEV}_{1}$ decline and an increased risk of hospitalization. ${ }^{1}$

Lack of awareness of nighttime respiratory symptoms can lead to worsened COPD control, disturbed sleep, and impaired daytime performance..$^{3-9}$ Current information about cough in COPD is based on subjective reporting. In most cases, cough is measured by using diary card scores. LEOSound lung sound monitor is a mobile system validated for automated long-term recording and objective analysis of normal and adventitious lung sounds like cough and wheezing. ${ }^{10-12}$ 
Several ways of quantifying cough have been published in recent years. ${ }^{13,14}$ Although, in practice, cough mostly occurs in epochs, most of the studies focus on cough frequency quantified by counting expulsive phases. ${ }^{15}$ Thus, the aims of this study were to employ a novel ambulatory system recording cough objectively and to look for severity, characteristics, and time distribution of nighttime cough events in different COPD stages.

\section{Materials and methods}

In the original observational study, we investigated prevalence and severity of cough and wheezing in 48 patients with stable COPD stages II-IV for two consecutive nights with the LEOSound system. ${ }^{16}$ All patients were recruited in an outpatient medical center, and the recordings were performed at the patient's home. Only patients who were free from current or recent exacerbations and from other acute or chronic respiratory diseases, such as asthma, were included in this study. To get equally sized and matched groups for each COPD stage, we randomly selected 30 out of the 48 patients, with similar age and body mass index. Nighttime cough was analyzed for frequency, severity, and pattern and is defined as cough taking place between bedtime and getting up in the morning. Our COPD subgroups of patients were between 51 and 79 years old with pack-years ranging from 0 to 90 . Eleven out of the 30 were still smoking at the time of recording. Demographics are presented in Table 1. The protocol was approved by the Marburg Ethics Committee before study initiation, and written informed consent was obtained from all patients.

\section{Acoustic long-term recording (LEOSound)}

Patients underwent two ambulant long-term recordings of nighttime cough and wheezing with the LEOSound system (Löwenstein Medical GmbH \& Co. KG, Bad Ems,

Table I Demographics, baseline characteristics, and lung function of the dataset

\begin{tabular}{llll}
\hline & $\begin{array}{l}\text { COPD II } \\
(\mathbf{n}=\mathbf{I 0})\end{array}$ & $\begin{array}{l}\text { COPD III } \\
(\mathbf{n}=\mathbf{l 0})\end{array}$ & $\begin{array}{l}\text { COPD IV } \\
(\mathbf{n}=\mathbf{I 0})\end{array}$ \\
\hline Age (years) & $65.8 \pm 7.2$ & $67.6 \pm 4.6$ & $63.8 \pm 8.6$ \\
BMI (kg/m $)$ & $26.9 \pm 6.4$ & $26.6 \pm 5.8$ & $23.3 \pm 4.6$ \\
Pack-years & $50.5 \pm 11.9$ & $45.4 \pm 21.6$ & $37.0 \pm 19.9$ \\
Male to female ratio & $5: 5$ & $6: 4$ & $5: 5$ \\
Severity according & $\mathrm{A}(\mathrm{n}=5)$ & $\mathrm{C}(\mathrm{n}=6)$ & $\mathrm{C}(\mathrm{n}=7)$ \\
to A/B/C/D & $\mathrm{B}(\mathrm{n}=5)$ & $\mathrm{D}(\mathrm{n}=4)$ & $\mathrm{D}(\mathrm{n}=3)$ \\
FEV $_{1}(\%)$ & $60.8 \pm 7.3$ & $44.0 \pm 8.7$ & $30.6 \pm 9.9$ \\
\hline
\end{tabular}

Note: Data presented as mean \pm standard deviation unless stated otherwise. Abbreviation: BMI, body mass index.
Germany). LEOSound is an automated lung sound monitor that continuously records lung sounds, such as wheezing and cough, by using three bioacoustical sensors placed at the back and trachea of the patient (Figure 1). To distinguish lung sounds from environment noise, the device is equipped with an internal ambient microphone. The system automatically detects cough and wheezing up to $24 \mathrm{~h}$ and can be used either in the hospital or at the patient's home. The automated detection algorithm for cough achieves a sensitivity of $93 \%$ and a specificity of $99 \% .^{12}$

\section{Cough definition and analysis}

The present study determined frequency, severity, and characteristics of cough during nighttime. Cough events were automatically marked by the system's analyzer software. Cough epochs were defined using the European Respiratory Society (ERS)'s definition, where cough epochs are classified as continuous coughing without a 2 s pause. ${ }^{13}$ Further, we distinguished two types of cough: productive (cough with audible mucus) and "normal" or nonproductive (cough without audible mucus). Each cough event was classified by trained medical experts whether it was productive or not, by listening to the characteristic cough sound. A previous investigation by Smith et al has shown the ability of health care professionals to distinguish between these types of cough with a high accuracy by listening solely to the sound of cough. ${ }^{17}$ If an epoch contained at least one productive cough event, the epoch was classified as productive. Furthermore, we investigated median cough epoch length and median number of cough events in a cough epoch.

\section{Statistics}

We tested the distribution of all cough variables for normality by using Kolmogorov-Smirnov test. For results with $p \leq 0.05$ we considered a significant deviation from normality. Because none of the variables showed normal distribution, we used nonparametric tests for interference statistics. Differences in groups were assessed by using Mann-Whitney $U$ test. Results were considered as significant with $p \leq 0.05$. To compare the groups, the average of both nights was taken. However, differences in groups should not be generalized due to the small number of patients included in this study.

\section{Results}

Cough events were found in all 30 patients ranging from 1 to 294 events over the recording period. Cough epochs could be found in 29 patients ranging from 1 to 75 . The highest frequency of cough epochs was found in patients 


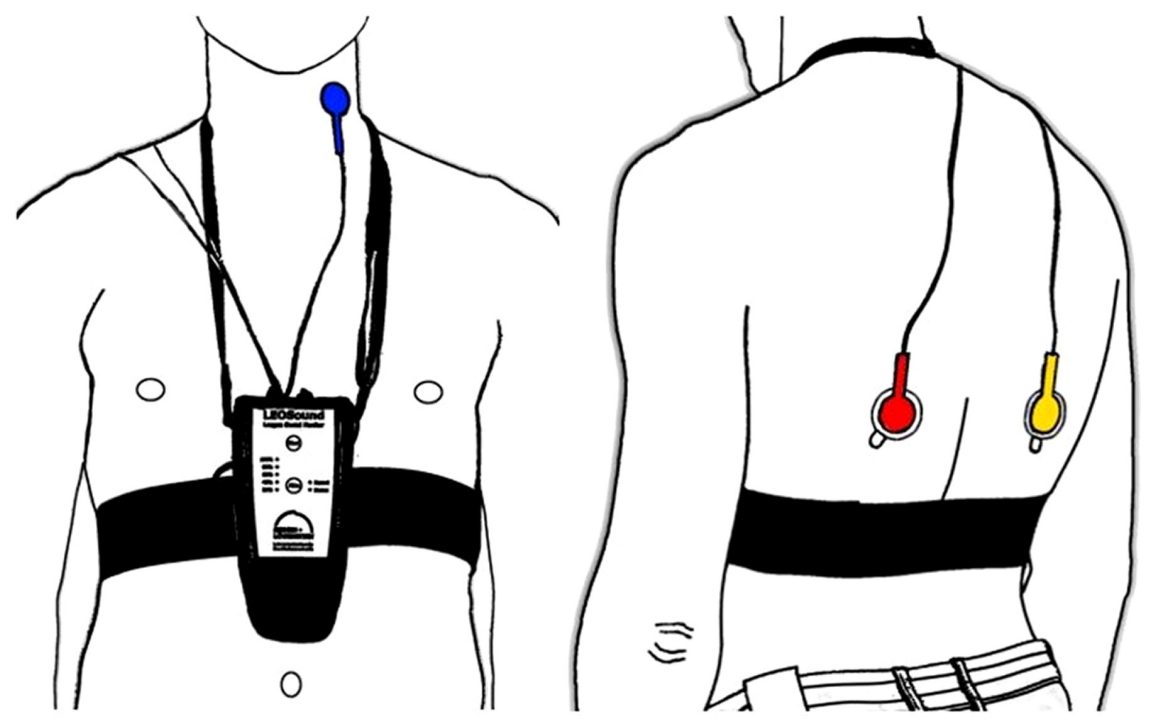

Figure I LEOSound recorder with connected trachea microphone (left) and two lung microphones (right).

with COPD stage III (Figure 2). Of all cough events, $75.3 \% \pm 23.3 \%$ occurred in cough epochs. Patients suffering from COPD II had the least amount of cough occurring in epochs $(62.6 \% \pm 28.4 \%)$, whereas in patients with COPD stages III and IV more than $80 \%$ of cough occurred in epochs $(80.9 \% \pm 16.7 \%$ and $82.5 \% \pm 18.7 \%$, respectively).

Figure $3 \mathrm{~A}-\mathrm{C}$ shows the distributions of productive and nonproductive cough epochs for the different COPD stages.

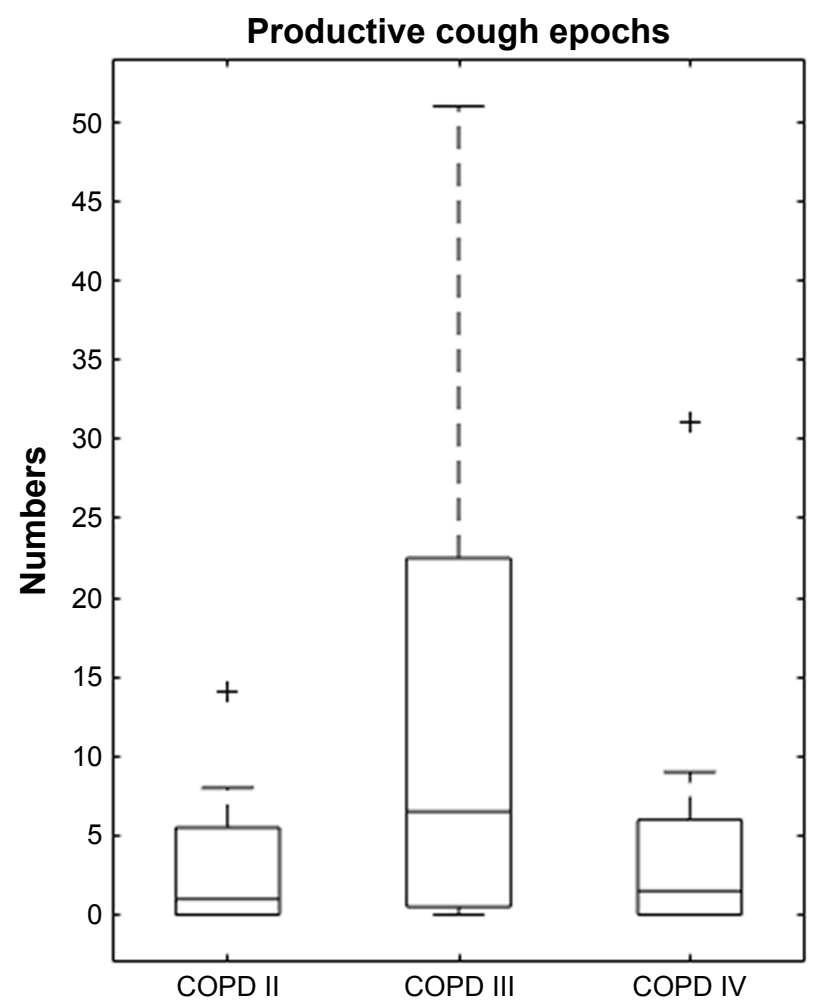

Both nights (marked as N1, N2) of each patient were divided into two equally sized halves (marked as H1, H2). The first half of each night contains a period where the patients are falling asleep followed by a sleeping period, while the second half mostly consists of sleep. There were no significant differences between the two halves. However, most of the patients had a similar distribution of cough epochs in both halves during each night, although the amount of cough epochs was

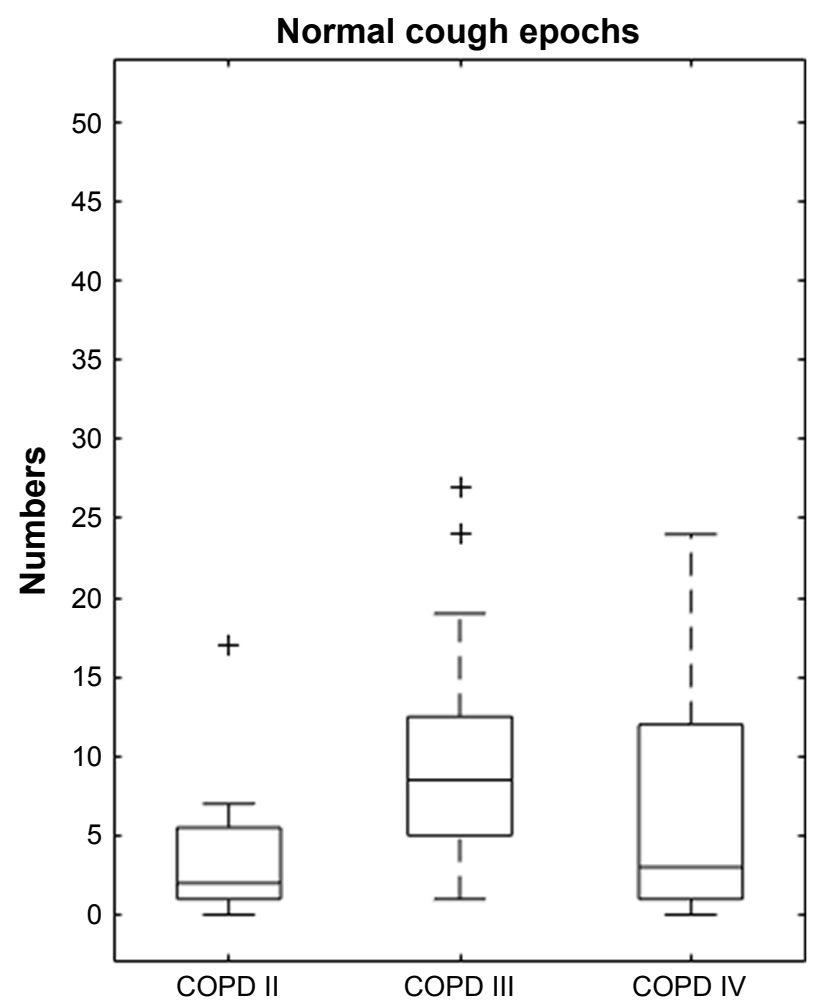

Figure 2 Distribution of productive and normal cough epochs grouped by COPD stage. 
not equal. There are also patients with different amounts and different distributions of cough epochs over both nights.

Within our COPD subgroups, parameters like median epoch length and median number of cough events in cough epochs do not show significant variations (Figures 4 and 5). Active smokers $(\mathrm{n}=11)$ showed more productive cough epochs (60.9\%) than nonsmokers $(\mathrm{n}=19,24.4 \%, p=0.0033$;
Figure 6). The mean pack-years for active smokers was 48.2 $( \pm 14.0)$ and for nonsmokers, it was $42.1( \pm 22.1, p=0.36)$.

\section{Discussion and conclusion}

Ambulatory cough recording by LEOSound offers a promising opportunity in the objective assessment of cough. We found a high rate of nighttime cough epochs in patients

A



B

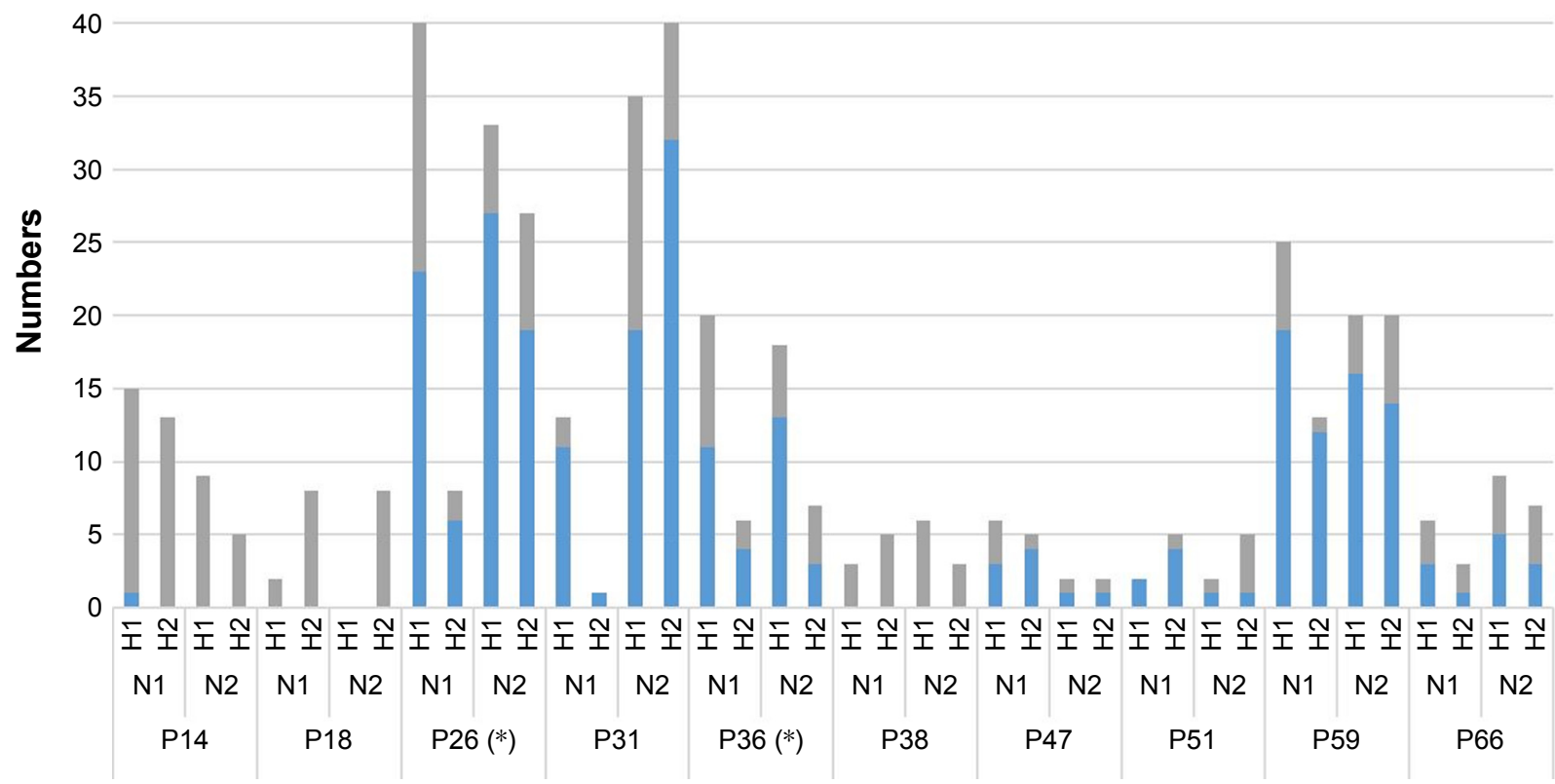

Figure 3 (Continued) 


\section{C}

45

COPD IV cough epochs



Figure 3 (A-C) Distribution of productive (blue) and normal (gray) cough epochs for all patients grouped by their COPD stage. Note: *Denotes smokers $(\mathrm{n}=\mathrm{I} \mathrm{I})$.

with COPD, especially in those with GOLD stage III. More than three-quarters of all cough events occurred in cough epochs. Productive cough was predominantly found in patients with persistent smoking. In active smokers, $60.9 \%$ of the cough epochs were productive, whereas it is $24.4 \%$ in nonsmokers.

Several ways of quantifying cough have been published in recent years. ${ }^{13,14}$ Cough sounds typically consist of three phases, an explosive one, an intermediate, and often, a voiced one. It is essential that cough monitoring devices define exactly what is recognized as cough and how cough

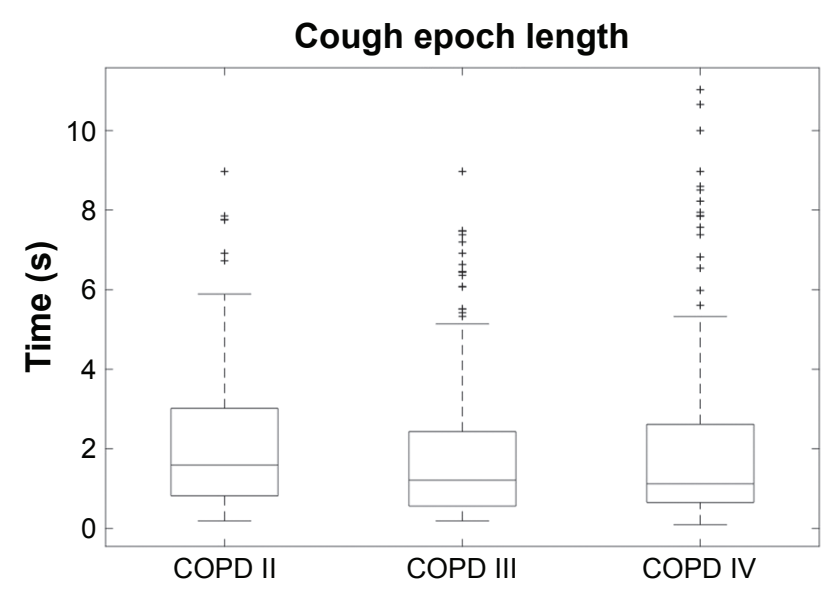

Figure 4 Distribution of cough length, grouped by COPD stage. is quantified. The explosive phase induces the characteristic sound of a single cough event. However, most cough events occur in bursts or epochs, and single coughs are not the typical pattern for patients with chronic respiratory diseases. In our study, more than $75 \%$ of cough events have been found as cough epochs. Using cough epochs to quantify coughing might represent the amount of cough inducing stimuli in a certain time period. The ERS defines a cough epoch as at least two consecutive cough events with a maximum distance of $2 \mathrm{~s} .{ }^{13}$ The results of our study support the statement of Fontana and Widdicombe that cough mostly occurs

\section{Number of cough events in a cough epoch}



Figure 5 Distribution of the number of coughs in a cough epoch, grouped by COPD stage. 




Figure 6 Distribution of productive and nonproductive cough in smokers $(n=I I)$ and nonsmokers $(n=19)$.

in epochs. ${ }^{15}$ Especially in COPD patients, where cough is most likely caused by mucus transport in small airways, it is recommended that cough epochs be considered in the assessment of cough.?

Cough is a frequent respiratory symptom, especially in COPD patients with moderate disease. During end-stage disease, breathlessness becomes the more important symptom and cough is less frequent. ${ }^{7}$ A cross-sectional study by von Hertzen et al has also shown that patients with mild to moderate COPD show more cough and sputum production than those with a severe disease. ${ }^{18}$ Our results confirm these findings. A possible explanation for patients suffering from COPD III having the highest amount of coughing could be that their cell metaplasia and hyperplasia have progressed further than in the earlier stages of the disease, whereas the muscle atrophy has not progressed as far as it does in the end-stage COPD. Respiratory symptoms are particularly disruptive during the nighttime and/or early morning, contributing to sleep disturbance, limited morning activities, and poor health status. ${ }^{2-4}$ Morning has been reported as the worst time of day especially among those patients with severe COPD. ${ }^{19-21}$ However, there are no objective data about the influence of cough on the sleep, presumably because of the lack of objective monitoring devices. Therefore, further investigations with automated, objective cough monitoring methods are necessary to show if cough causes sleep arousals.

Lack of validated and objective measures of cough frequency and severity has hampered understanding cough and its relationship with different respiratory diseases. ${ }^{13}$ Up to now assessment of cough severity relies on subjective methods. Visual analog scales, questionnaires, and self-report coughing diary cards have been used, but these do not directly correlate with objective methods such as ambulatory cough monitoring. Subjective assessments, such as scales or questionnaires, give physicians a different perspective on the symptom cough, especially when quality of life is affected by a lot more than just the amount of coughing. Therefore, international experts recommend using both subjective and objective methods when investigating cough. ${ }^{14}$ The original observational study also collected the COPD Assessment Test data, but no data about the quality of life of the patients. These data should be considered in future studies.

Nighttime symptoms are those between the time of going to bed and the time of getting up to start the day; morning symptoms are defined as those between the time of getting out of bed and 11:00 am. Most of the cough periods in our patients were nonproductive during nighttime. This correlates with the fact that productive cough is predominantly found in the morning hours after getting up from bed. ${ }^{19}$ Clearance of airway mucus after sleep may explain the increased cough frequency in the first hours after wakening. To understand the underlying processes, this crucial time in the morning needs to be investigated, with both subjective and objective assessments of cough, in more detail. Productive cough being strongest both during sleep and the morning hours is highly suggestive of still smoking COPD patients. This was also true for our patients. The 11 active smokers had significantly more productive cough epochs (61\%) than the nonsmokers (24\%). The groups of smokers and nonsmokers in our study are comparable in terms of pack-years. Because mean pack-years in both groups differed in just a small way, it is very likely that continued tobacco exposure leads to more productive cough. Therefore, we come to a conclusion similar to other 
researchers that persistent smoking is the most important risk factor for cough and COPD progression. ${ }^{1,19,22,23}$ In chronic smokers, impaired mucus clearance leads to chronic irritation of airway epithelium and promotes cellular metaplasia. Nonsmokers with COPD report less chronic cough and sputum and have less impairment in airflow limitation and gas exchange. ${ }^{8}$

On closer inspection of cough epochs, our patients show individual pattern of cough distribution (N1, N2 and H1, H2), which might be considered in future investigations. Parameters like median epoch length and median number of cough events in a cough epoch did not vary in our subgroups, but might also be interesting for future cough studies, especially when acute diseases are investigated.

In summary, LEOSound lung sound monitor offers a good chance to evaluate (nighttime) cough, which might otherwise stay unnoticed. Cough during sleep is frequent, and most of the cough events occur in cough epochs. The potential drivers for chronic cough in COPD are multifactorial. Cigarette smoke exposure is the most important factor for productive cough. Systematic 24-hour studies with ambulatory monitoring of chronic cough in larger numbers of well-defined COPD patients are necessary to characterize cough exactly. Although findings of this study cannot be generalized, they give a first impression on what is happening with coughs in COPD during the nighttime. Further investigations containing objective cough data are necessary to reproduce our findings and get a better understanding of cough in COPD.

\section{Acknowledgments}

This study was funded by GlaxoSmithKline plc, Brentford, UK. Preliminary results of this study have been presented at the ATS 2017 International Conference (May 19-24) in Washington, DC, USA. The abstract can be found using the following link: http://www.atsjournals.org/doi/abs/10.1164/ ajrccm-conference.2017.195.1_MeetingAbstracts.A2684.

\section{Author contributions}

PF analyzed the data, designed the concept of the article, and critically evaluated the manuscript. VG and KS analyzed the data and critically evaluated the manuscript. JK carried out the measurements and critically evaluated the manuscript. AW designed the concept of the study and critically evaluated the manuscript. OH designed the concept of the study, prepared the data, and critically evaluated the manuscript. UK designed the concept of the study, the concept of the article, and critically evaluated the manuscript. All the authors approved the final manuscript as submitted.

\section{Disclosure}

Ulrich Koehler is affiliated with Löwenstein Medical GmbH \& Co. KG, IfM, GlaxoSmithKline, Resmed, UCB Pharma Boehringer Ingelheim, and Sanofi. Patrick Fischer, Volker Gross, and Andreas Weissflog are affiliated with Sanofi. The other authors report no conflicts of interest in this work.

\section{References}

1. Vestbo J, Prescott E, Lange P. Association of chronic mucus hypersecretion with FEV1 decline and chronic obstructive pulmonary disease morbidity. Copenhagen City Heart Study Group. Am J Respir Crit Care Med. 1996;153(5):1530-1535.

2. Soler-Cataluña JJ, Sauleda J, Valdés L, et al. Prevalence and perception of 24-hour symptom patterns in patients with stable chronic obstructive pulmonary disease in Spain. Arch Bronconeumol. 2016;52(6):308-315. Spanish.

3. Agusti A, Hedner J, Marin JM, Barbé F, Cazzola M, Rennard S. Nighttime symptoms: a forgotten dimension of COPD. Eur Respir Rev. 2011; 20(121):183-194.

4. Lange P, Marott JL, Vestbo J, Nordestgaard BG. Prevalence of nighttime dyspnoea in COPD and its implications for prognosis. Eur Respir J. 2014;43(6):1590-1598.

5. Roche N, Chavannes NH, Miravitlles M. COPD symptoms in the morning: impact, evaluation and management. Respir Res. 2013; 14(1): 112 .

6. Miravitlles M, Ribera A. Understanding the impact of symptoms on the burden of COPD. Respir Res. 2017;18(1):67.

7. Smith J, Woodcock A. Cough and its importance in COPD. Int J Chron Obstruct Pulmon Dis. 2006;1(3):305-314.

8. Calverley PM. Cough in chronic obstructive pulmonary disease: is it important and what are the effects of treatment? Cough. 2013;9:17.

9. Zhang J, Lin X, Bai CX. Comparison of clinical features between nonsmokers with COPD and smokers with COPD: a retrospective observational study. Int J Chron Obstruct Pulmon Dis. 2014;9:57-63.

10. Koehler U, Brandenburg U, Weissflog A, Sohrabi K, Groß V. [LEOSound, an innovative procedure for acoustic long-term monitoring of asthma symptoms (wheezing and coughing) in children and adults]. Pneumologie. 2014;68(4):277-281. German.

11. Gross V, Reinke C, Dette F, et al. Mobile nocturnal long-term monitoring of wheezing and cough. Biomed Tech (Berl). 2007;52(1):73-76.

12. Gross V, Scholtes M, Sohrabi K, Weissflog A, Nikolaizik W, Koehler U. Validation of the LEOSound ${ }^{\mathbb{R}}$-monitor for standardized detection of cough and wheezing in children. In: Abstract Book. Istanbul; 2015:143. Available from: http://www.congrex-switzerland.com/fileadmin/ files/2015/worldsleep2015/pdf/Worldsleep_2015_-_Online_Abstract_ Book_by_session_date_and_time.pdf. Accessed August 29, 2017.

13. Morice AH, Fontana GA, Belvisi MG, et al. ERS guidelines on the assessment of cough. Eur Respir J. 2007;29(6):1256-1276.

14. Kelsall A, Decalmer S, Webster D, et al. How to quantify coughing: correlations with quality of life in chronic cough. Eur Respir J. 2008; 32(1):175-179.

15. Widdicombe J, Fontana G. Cough: what's in a name? Eur Respir J. 2006; 28(1):10-15.

16. Krönig J, Hildebrandt O, Weissflog A, et al. Long-term recording of night-time respiratory symptoms in patients with stable COPD II-IV. COPD. 2017;14(5):498-503.

17. Smith JA, Ashurst HL, Jack S, Woodcock AA, Earis JE. The description of cough sounds by healthcare professionals. Cough. 2006;2:1.

18. von Hertzen L, Reunanen A, Impivaara O, Mälkiä E, Aromaa A. Airway obstruction in relation to symptoms in chronic respiratory disease-a nationally representative population study. Respir Med. 2000; 94(4):356-363.

19. Sumner H, Woodcock A, Kolsum U, et al. Predictors of objective cough frequency in chronic obstructive pulmonary disease. Am J Respir Crit Care Med. 2013;187(9):943-949. 
20. Tsiligianni I, Metting E, van der Molen T, Chavannes N, Kocks J. Morning and night symptoms in primary care COPD patients: a crosssectional and longitudinal study. An UNLOCK study from the IPCRG. NPJ Prim Care Respir Med. 2016;26:16040.

21. Stephenson JJ, Cai Q, Mocarski M, Tan H, Doshi JA, Sullivan SD. Impact and factors associated with nighttime and early morning symptoms among patients with chronic obstructive pulmonary disease. Int J Chron Obstruct Pulmon Dis. 2015;10:577-586.
22. Ramos FL, Krahnke JS, Kim V. Clinical issues of mucus accumulation in COPD. Int J Chron Obstruct Pulmon Dis. 2014;9:139-150.

23. Martin MJ, Harrison TW. Causes of chronic productive cough: an approach to management. Respir Med. 2015;109(9):1105-1113.

\section{Publish your work in this journal}

The International Journal of COPD is an international, peer-reviewed journal of therapeutics and pharmacology focusing on concise rapid reporting of clinical studies and reviews in COPD. Special focus is given to the pathophysiological processes underlying the disease, intervention programs, patient focused education, and self management protocols.

\section{Dovepress}

This journal is indexed on PubMed Central, MedLine and CAS. The manuscript management system is completely online and includes a very quick and fair peer-review system, which is all easy to use. Visit http://www.dovepress.com/testimonials.php to read real quotes from published authors. 